\title{
Contribution of Horizontal Gene Transfer and Deletion Events to Development of Distinctive Patterns of Fimbrial Operons during Evolution of Salmonella Serotypes
}

\author{
ANDREAS J. BÄUMLER, ${ }^{1,2 *}$ ANDRIES J. GILDE,${ }^{1}$ RENÉE M. TSOLIS, ${ }^{1,3}$ ADRIANUS W. M. VAN DER VELDEN, \\ BRIAN M. M. AHMER, ${ }^{1}$ AND FRED HEFFRON ${ }^{1}$ \\ Department of Molecular Microbiology and Immunology, Oregon Health Sciences University, Portland, Oregon 97201-3098 ${ }^{1}$; \\ Department of Medical Microbiology \& Immunology, Texas A\&M University, College Station, Texas 77843-1114²; \\ and Department of Veterinary Pathobiology, Texas A\&M University, College Station, Texas 77843-44673
}

Received 21 August 1996/Accepted 23 October 1996

\begin{abstract}
Only certain serotypes of Salmonella represent $99 \%$ of all human clinical isolates. We determined whether the phylogenetic distribution of fimbrial operons would account for the host adaptations observed for Salmonella serotypes. We found that three fimbrial operons, $\mathrm{fim}$, lpf, and $a g f$, were present in a lineage ancestral to Salmonella. While the fim and agf fimbrial operons were highly conserved among all Salmonella serotypes, sequence analysis suggested that the lpf operon was lost from many distantly related lineages. As a consequence, the distribution of the $l p f$ operon cannot be explained easily and may be a consequence of positive and negative selection in different hosts for the presence of these genes. Two other fimbrial operons, sef and pef, each entered two distantly related Salmonella lineages and each is present only in a small number of serotypes. These results show that horizontal gene transfer and deletion events have created unique combinations of fimbrial operons among Salmonella serotypes. The presence of sef and pef correlated with serotypes frequently isolated from common domesticated animals.
\end{abstract}

The genus Salmonella and the species Escherichia coli are phylogenetically closely related, diverging from a common ancestor between 100 and 160 million years ago $(21,40)$. The gene order on the chromosome has been highly conserved between these two organisms (45), and corresponding genes have an average of $84 \%$ sequence identity (50). These properties make $E$. coli and the genus Salmonella a unique pair with which to identify evolutionary changes in genomes. A comparison of the genetic map of E. coli K-12 with that of serotype Salmonella typhimurium, which identified some 30 large DNA segments present only in one of these organisms, provided the first evidence that horizontal gene transfer mediated by plasmids or phage was a major driving force during the evolution of these bacteria (45). The genetic material received by the genus Salmonella by way of horizontal gene transfer includes several segments encoding virulence factors. The transferred DNA may vary in size from large genetic elements, such as the pathogenicity islands SPI 1 (34) and SPI 2 (51), phage (38), and virulence plasmids (27), to fimbrial operons located on the chromosome (5), or even single genes inserted in a housekeeping operon (52). Although acquisition of these virulence genes has significantly influenced the evolution of Salmonella serotypes, little is known about their ancestry. The first step in understanding how these virulence factors contributed to shape this successful pathogen is to determine the point in evolution when they were acquired.

The classification of the genus Salmonella into serotypes according to the Kauffmann and White scheme is not particularly useful for evolutionary studies since it is based on three surface markers and therefore does not provide a good basis for estimating genetic relatedness among strains (43). The

\footnotetext{
* Corresponding author. Mailing address: Department of Medical Microbiology \& Immunology, 407 Reynolds Medical Building, Texas A\&M University, College Station, TX 77843-1114. Phone: (409) 8627756. Fax: (409) 845-3479.
}

inherent weakness of this system was pointed out by Selander and coworkers, who showed by multilocus enzyme electrophoresis (MLEE) that many Salmonella serotypes are not of monophyletic origin $(8,49)$. An analysis of DNA relatedness $(20,30)$, MLEE $(10,44)$, and sequence analysis $(9,32,36,37)$ established taxonomic schemes which reflect the actual phylogenetic relationships among Salmonella serotypes. According to this current nomenclature the genus Salmonella contains only two species, Salmonella bongori (44) and Salmonella enterica (29), the latter of which is further subdivided into subspecies designated by roman numerals. Thus, the antigen formulas of the Kauffmann and White scheme describe serotypes rather than species (e.g., S. enterica serotype Typhimurium, although Latin binomials for serotypes are still in use for practical reasons, e.g., S. typhimurium). This differentiation into species and subspecies involved changes in the host range of Salmonella serotypes. For example, S. enterica subspecies I is predominantly isolated from warm-blooded animals, whereas $S$. bongori and the other subspecies of $S$. enterica are most frequently isolated from reptiles $(12,43,56)$. The importance of these changes in host range is highlighted by the fact that $99 \%$ of clinical isolates belong to $S$. enterica subspecies I (43). What are the genetic alterations that allowed $S$. enterica subspecies I to change its host range to include warm-blooded animals as hosts? While fimbriae have been shown to determine the host range of enteropathogenic E. coli $(25,28,42)$, little is known about the virulence factors involved in determining host specificity of Salmonella serotypes. Several fimbrial operons, including fim (14), lpf (5), pef (26), sef (16), and agf (22), have been identified in Salmonella serotypes. Did horizontal transfer of these fimbrial operons play a role during the evolution of $S$. enterica subspecies I? To answer these questions, we determined the distribution of fimbrial operons among two collections of Salmonella serotypes with known phylogenetic relationships $(10,44)$. 


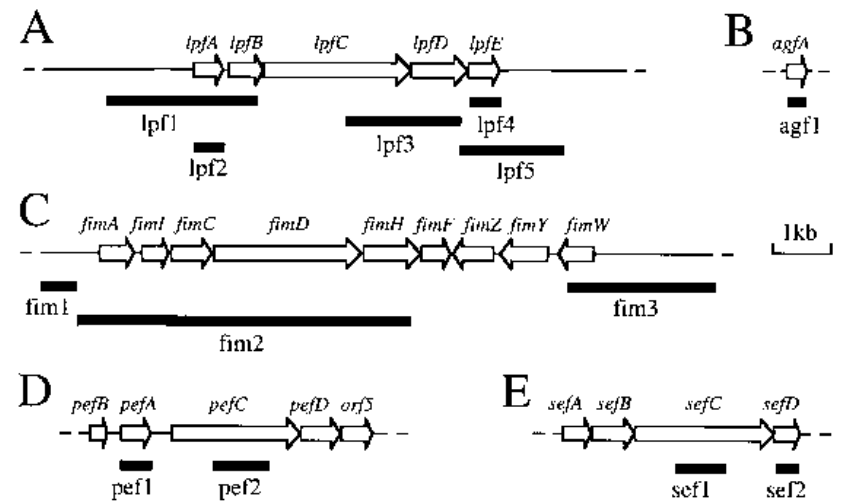

FIG. 1. Positions of DNA fragments (black bars) used as probes for detection of fimbrial operons. The positions of genes within the lpf (A), agf (B), fim (C), pef (D), and sef (E) fimbrial operons are indicated by arrows.

\section{MATERIALS AND METHODS}

Bacterial strains. Salmonella reference collection B (SARB) has been reported on recently (10). A collection of Salmonella serotypes representing $S$. bongori and six subspecies of $S$. enterica has been described by Reeves et al. (44). E. coli strains TA One Shot and DH5 $\alpha$ were purchased from Invitrogen and Gibco BRL, respectively.

PCR and generation of nucleotide probes. The primers for PCR amplification of a region between open reading frame 103 (orf103) and orf101 in E. coli and the corresponding regions on the chromosomes of Salmonella serotypes were 5'-CAATGACAATAATATCGCCGAATTCGGCTC-3' and 5'-CGGCCAAAC AGTGAAAGAAGACG-3'. Amplification was achieved by 25 cycles of amplification with $\mathrm{Taq}$ polymerase, the conditions for each cycle being $95^{\circ} \mathrm{C}$ for $1 \mathrm{~min}$, $50^{\circ} \mathrm{C}$ for $1 \mathrm{~min}$, and $72^{\circ} \mathrm{C}$ for $6 \mathrm{~min}$. PCR products were cloned into the vector pCRII and transformed into the E. coli strain TA One Shot (TA cloning kit; Invitrogen). To generate nucleotide probes, DNA fragments were labeled with the DNA labeling and detection kit (nonradioactive) from Boehringer Mannheim. Plasmid pISF101 (13) was digested with HpaI-SphI, and probes fim1, fim2, and fim 3 were generated by labeling the restriction fragments depicted in Fig. 1. The inserts of plasmids pMS1067, pMS1039, and pMS1048 (5) were labeled to produce probes $\operatorname{lpf} 1, \mathrm{lpf} 3$, and lpf5, respectively. Primers 5'-TTGCTCTGTCTG CTCTCGCTGTAG-3' and 5'-CATGATTCTCTTCCTGAGCCTCC-3' were used for PCR amplification of the lpfA gene from serotype $S$. typhimurium (Fig. 1). Primers for amplification of $l p f E$ were $5^{\prime}$-TTTGATGCCAGCGTGTTTACT G-3' and 5'-AGTAGACCACCAGCAGAGGGAAAG-3' (Fig. 1). The pef $A$ specific nucleotide probe pef1 has been described previously (6). The insert of plasmid pPE1 (6) was labeled to generate probe pef2. A 1,103-bp internal fragment of $\operatorname{sefC}$ was amplified by PCR from Salmonella enteritidis En1 with primers 5'-GCGAAAACCAATGCGACTGTAG-3' and 5'-CCCACCAGAAA CATTCATCCC-3' (Fig. 1). Primers 5'-TCAACTATTAAAGCACAAGAAC-3'

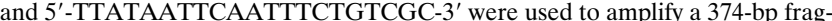
ment of sefD from serotype $S$. enteritidis En1 (Fig. 1). A 151-bp fragment of agf $A$ from serotype $S$. typhimurium was amplified with primers 5 '-TGCAAAGCGAT GCCCGTAAATC-3' and 5'-TTAGCGTTCCACTGGTCGATGGTG-3' (Fig. $1)$.

Southern hybridization. Isolation of chromosomal DNA was performed as recently described (3). Chromosomal DNA was restricted with EcoRI, and the fragments were separated on a $0.5 \%$ agarose gel. Southern transfer of DNA onto a nylon membrane was performed as previously described (3). Hybridization was performed at $65^{\circ} \mathrm{C}$ in solutions without formamide. Two 15 -min washes were performed under nonstringent conditions at room temperature in $2 \times$ SSC (1× SSC is $0.15 \mathrm{M} \mathrm{NaCl}$ plus $0.015 \mathrm{M}$ sodium citrate) $-0.1 \%$ sodium dodecyl sulfate. Hybrids were detected with the labeling and detection kit (nonradioactive) from Boehringer Mannheim.

Nucleotide sequencing. To compensate for PCR errors, products from two independent reactions were cloned in each case, and sequencing was performed by the dideoxy chain termination method (47) with an AutoRead sequencing kit (Pharmacia) and an ALF automatic sequenator. The nucleotide sequences were analyzed with programs blastX and blastN (1).

\section{RESULTS}

Distribution of fimbrial operons among strains of $S$. enterica subspecies I. The frequencies of homologs of the lpf and pef operons have not been established within Salmonella serotypes $(5,6)$. The distributions of the agf $(22)$, sef $(15,54)$, and fim operons (53) have produced some very useful epidemiological data. However, strains of the same serotype might be only distantly related in genotype $(8,49)$, and hence these earlier reports provided little information about the evolutionary origins of fimbrial operons. To obtain clues as to when during evolution fimbrial operons entered the gene pool of the genus Salmonella, we determined their distribution by using the SARB collection, which includes 72 strains representing 37 serotypes of $S$. enterica subspecies I (10). Hybridization with probes fim1, fim2, fim3, and agf1 (Fig. 1) revealed that the fim and agf fimbrial operons were acquired in a lineage ancestral to S. enterica subspecies I. DNA probes lpf2, lpf3, and lpf4 (Fig. 1) each produced identical hybridization patterns (Fig. 2). These hybridization patterns may best be explained by repeated loss of the lpf operon, although their generation by repeated acquisition of $l p f$ genes cannot be ruled out. Probes lpf1 and lpf5 contain sequences upstream and downstream of the lpf operon; these sequences are also present at the corresponding location in the $E$. coli chromosome (5). These probes detected homologous sequences in all strains of the SARB collection, indicating that a putative repeated loss of the lpf operon never involved large deletions of upstream or downstream sequences (Fig. 2). Probes sef1 and sef2 (Fig. 1) both hybridized with chromosomal DNA of two phylogenetic lineages (Fig. 2), suggesting recent acquisition by two lateral transfer events. Further support for this idea comes from the atypical base composition of sef. The $\mathrm{G}+\mathrm{C}$ content, which in the case of $S$. enterica averages $52 \%$, is considered to be a phylogenetic characteristic of a species (2). However, the genes sef $A B C D$ have a $\mathrm{G}+\mathrm{C}$ content of only $35.2 \%(15,16)$, which suggests that this operon was obtained horizontally from an organism with low $\mathrm{G}+\mathrm{C}$ content. Probes pef1 and pef2 (Fig. 1) detected signals in two distantly related lineages within $S$. enterica subspecies I (Fig. 2). The restricted phylogenetic distribution of pef is evidence for its recent acquisition by horizontal transfer, in fact, by a plasmid-mediated event, as this operon was recently located on the virulence plasmid (26). The reported variability among virulence plasmids isolated from different Salmonella serotypes $(11,35)$ may explain why probes pef1 and pef 2 did not produce perfectly matching hybridization patterns.

Distribution of fimbrial operons among $S$. bongori and $S$. enterica. Since $99 \%$ of clinical isolates belong to $S$. enterica subspecies I (43), previous studies on the distribution of fimbrial operons among Salmonella serotypes did not include a representative set of strains from $S$. bongori or other $S$. enterica subspecies $(22,53,54)$. To obtain information on the distribution of fimbrial operons among $S$. bongori and $S$. enterica subspecies II, IIIa, IIIb, IV, and VI, we used a set of 18 strains described by Reeves and coworkers (44). Probe agf1 detected homologs in $S$. bongori and in six subspecies of $S$. enterica (Fig. 3). This finding is consistent with the idea that agf $A$ was present in a common ancestor of all Salmonella serotypes. CsgA, the major fimbrial subunit of Curli in E. coli, has $74 \%$ amino acid sequence identity with AgfA (17), which is in the range of sequence conservation found between proteins present in $E$. coli and $S$. typhimurium (50). Since sequences related to agf $A$ were not detected in enterobacteria more distantly related to the genus Salmonella (22), it is likely that the agf operon was acquired by a common ancestor of E. coli and the genus Salmonella. Probes fim1, fim2, and fim3 (Fig. 1) detected homologs in all Salmonella serotypes tested, indicating that the fim operon entered a lineage ancestral to the genus Salmonella (Fig. 3). The fim operon has been mapped to $14 \mathrm{~min}$ on the $S$. typhimurium LT-2 genetic map (18), while genes encoding type 1 fimbriae of $E$. coli $\mathrm{K}-12$ are located at 98 min (4). Differences in gene order and nucleotide sequence, as well as the different 


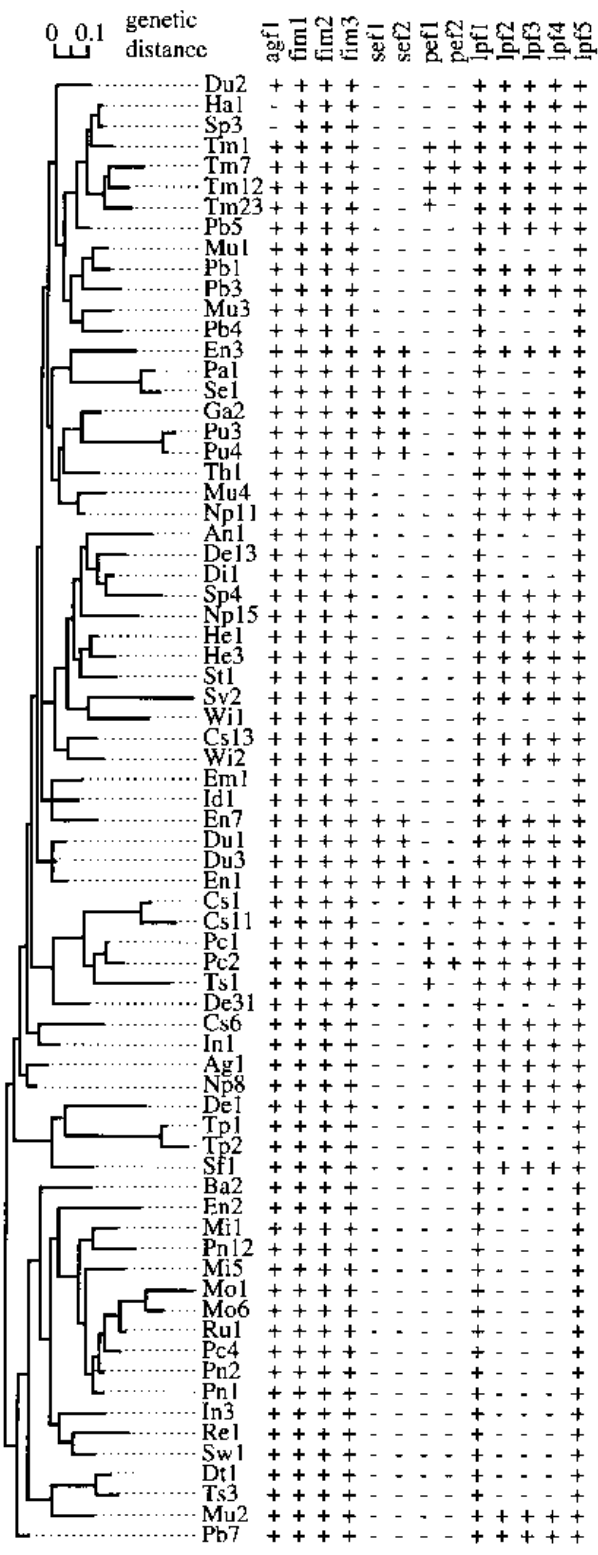

FIG. 2. Distribution of fimbrial operons among strains of $S$. enterica subspecies I (SARB collection). The left side shows the phylogenetic tree constructed by Boyd and coworkers (10). The distribution of fimbrial operons is shown on the right. Salmonella serotype abbreviations are as follows: Ag, S. agona; An, S. anatum; Ba, S. brandenburg; Cs, S. choleraesuis; De, S. derby; Di, S. duisburg; Dt, $S$. decatur; Du, S. dublin; Em, S. emek; En, S. enteritidis; Ga, S. gallinarum; Ha, $S$. haifa; He, S. heidelberg; Id, S. indiana; In, S. infantis; Mi, S. miami; Mo, S. montevideo; $\mathrm{Mu}, S$. muenchen; $\mathrm{Np}, S$. newport $\mathrm{Pa}, S$. paratyphi $\mathrm{A} ; \mathrm{Pb}, S$. paratyphi B; Pc, S. paratyphi C; Pn, S. panama; Pu, S. pullorum; Re, S. reading; Ru, $S$. rubislaw; Se, S. sendai; Sf, S. senftenberg; Sp, S. saintpaul; St, S. stanley; Sv, S. stanleyville; Sw, S. schwarzengrund; Th, S. thompson; Tm, S. typhimurium; Tp, $S$. typhi; Ts, S. typhisuis; Wi, S. wien. +, hybridization signal; - , no hybridization signal.

map positions of these fimbrial operons on otherwise colinear chromosomes (45), imply that they have distinct origins and were acquired independently. Probes lpf2, lpf3, and lpf4 (Fig. 1) gave hybridization signals with serotypes from $S$. bongori but did not detect any homologs in $S$. enterica subspecies II, IIIa, IIIb, IV, or VI. This distribution may be explained by acquisition in a lineage ancestral to the genus Salmonella and subse-

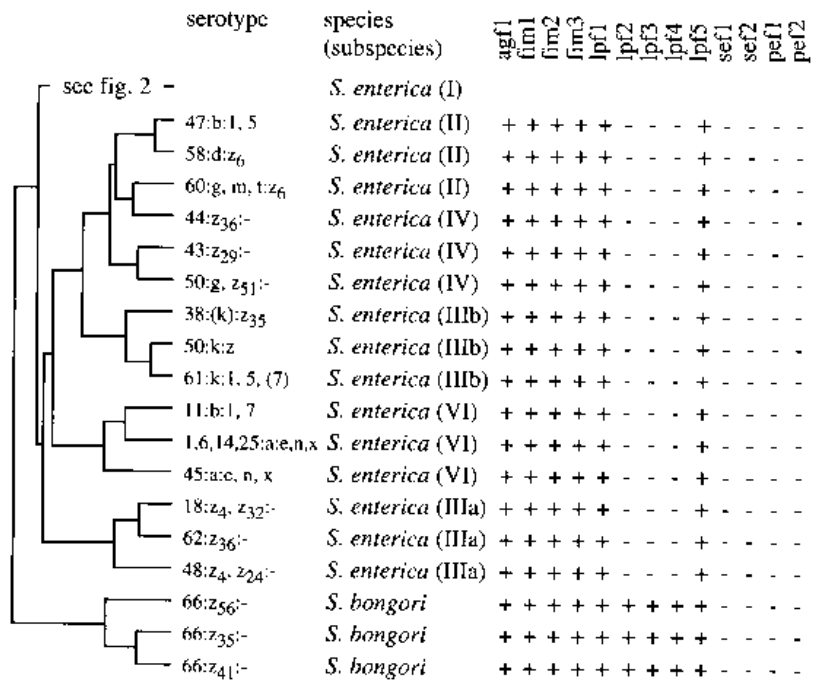

FIG. 3. Distribution of fimbrial operons among stains of $S$. bongori and $S$. enterica. The left side shows the phylogenetic tree constructed by Reeves and coworkers (44). The distribution of fimbrial operons is shown on the right. +, hybridization signal; -, no hybridization signal.

quent loss in a lineage ancestral to $S$. enterica subspecies II, IIIa, IIIb, IV, and VI (Fig. 3).

Comparison of the chromosomal regions upstream and downstream of $\operatorname{lpf}$ from $S$. typhimurium with corresponding regions in $E$. coli and different Salmonella serotypes. The genes lpf $A B C D E$ are located at $78 \mathrm{~min}$ on the S. typhimurium chromosome, and no homologs are present at the corresponding region of the E. coli K-12 chromosome. A sequence comparison revealed that these five genes are inserted between homologs of E. coli orf103 and orf102 at 78 min on the $S$. typhimurium chromosome (5). The complex phylogenetic distribution of the $l p f$ operon among serotypes of $S$. bongori and $S$. enterica is indicative of repeated acquisition and loss. To clarify this point we analyzed the chromosomal areas surrounding the lpf operon in Salmonella serotypes lacking these genes by PCR amplification. A comparison of the nucleotide sequences from $E$. coli K-12 (GenBank accession number U00039) and S. typhimurium (5) identified conserved nucleotide sequences for the design of primers which anneal downstream of lpfE (within orf102 of E. coli) and upstream of lpfA (upstream of orf104 of E. coli) (Fig. 4A). With this primer pair, no product was obtained for Salmonella serotypes which contain lpf, since the conditions used for PCR did not allow amplification of the entire 7-kb region. However, all Salmonella serotypes lacking lpf yielded PCR products of a single size (about $1.2 \mathrm{~kb}$ ). PCR amplification of the corresponding region from E. coli $\mathrm{DH} 5 \alpha$ yielded a product of about $2.7 \mathrm{~kb}$, the size predicted by the nucleotide sequence.

Further nucleotide sequence analysis of the $\operatorname{lpf} A$ upstream region showed that a $5^{\prime}$-truncated remnant of E. coli orf103 (94 bp of the $3^{\prime}$ end of orf103) was present in S. typhimurium (Fig. 4C). As compared to E. coli K-12, most of orf103 has been lost in $S$. typhimurium by a deletion of about 1,240 bp (Fig. 4B). It is not clear from these data whether this deletion occurred before (as shown in Fig. 4), after, or at the same time as the lpf operon entered the genome. The lpf operon has been inserted directly behind the stop codon of the $5^{\prime}$-truncated orf103 homolog in S. typhimurium (Fig. 4C). The PCR products obtained from three serotypes, Salmonella paratyphi A enzyme type Pal, Salmonella typhi enzyme type Tp2 and Salmonella II 


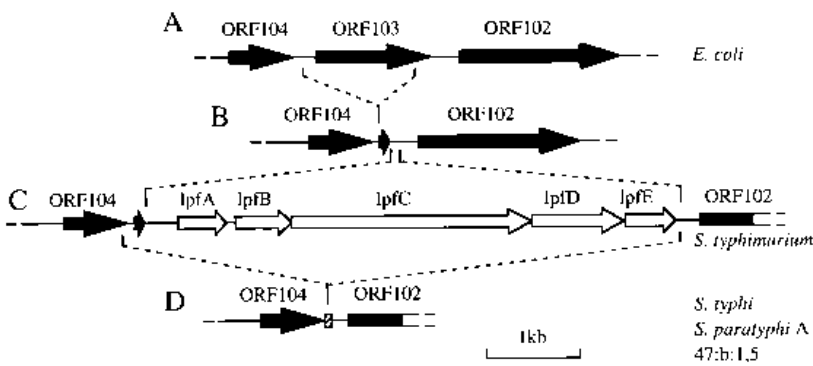

FIG. 4. Model for acquisition and loss of the lpf fimbrial operon. The region on the $S$. typhimurium chromosome carrying the lpf operon (C) and the corresponding regions from $S$. typhi, S. paratyphi A, serotype 47:b:1,5 (D), and E. coli (A) are shown. The positions of deletions or insertions are indicated by dashed lines. A hypothetical intermediate in these events is also shown (B). Genes with homology to those of E. coli are shown as black arrows. The position of a 78-bp sequence found only in $S$. typhi, S. paratyphi A, and serotype 47:b:1,5 is indicated by a hatched bar.

serotype 47:b:1,5, lacking $l p f$ were cloned into vector pCR II to produce plasmids pTY990, pTY989, and pTY991, respectively. Nucleotide sequencing revealed that the 5'-truncated remnant of orf103 was not present in these serotypes (Fig. 4D). A comparison with the $S$. typhimurium sequence showed a 6,057-bp deletion in $S$. typhi, $S$. paratyphi A, and serotype 47:b:1,5 which encompassed the 5 'truncated remnant of orf103 and the lpf operon (Fig. 4C, D). These findings imply that the lpf operon was first acquired by a lineage ancestral to the genus Salmonella and was subsequently deleted together with the 5'-truncated remnant of orf103 from S. typhi, S. paratyphi A, and serotype 47:b:1,5. The absence of orf103 also explains why the PCR products from Salmonella serotypes which lack the lpf operon were smaller than that obtained from E. coli K-12. The branching orders on the phylogenetic tree suggest that loss of the lpf operon from distantly related serotypes such as $S$. typhi, $S$. paratyphi A, and 47:b:1,5 occurred by independent events (Fig. 2, 3).

Interestingly, the deletion of the lpf operon in S. paratyphi A, S. typhi, and serotype 47:b:1,5 was accompanied by an insertion of $78 \mathrm{bp}$ in each case (Fig. 5). This sequence showed no homology to any entries in current nucleotide databases; however, it was highly conserved among $S$. paratyphi A, S. typhi, and serotype 47:b:1,5. The identity of the deletion endpoints and the simultaneous acquisition of a conserved 78-bp nucleotide sequence imply that the loss of lpf in S. typhi, S. paratyphi A, and serotype 47:b:1,5 involved a common mechanism, presumably horizontal transfer following a single ancestral deletion event. The phylogeny of Salmonella serotypes suggests that the lpf operon was initially lost from a lineage ancestral to S. enterica subspecies II, IIIa, IIIb, IV, and VI. This deletion of lpf may then have been transferred into other serotypes by homologous exchange of horizontally transferred segments. Our finding that PCR products of identical size were obtained for all Salmonella serotypes which lack the lpf operon is consistent with this idea. Similar evolutionary mechanisms result in genetic transfer events which generate allelic variation at the $f i C$ locus, thereby increasing serovar diversity within the genus Salmonella (31).

\section{DISCUSSION}

The phylogenetic relationships among Salmonella serotypes have been reconstructed by comparison of nucleotide sequences from homologous genes or by MLEE of homologous gene products $(8,9,36,37,44,49)$. These studies have pro-

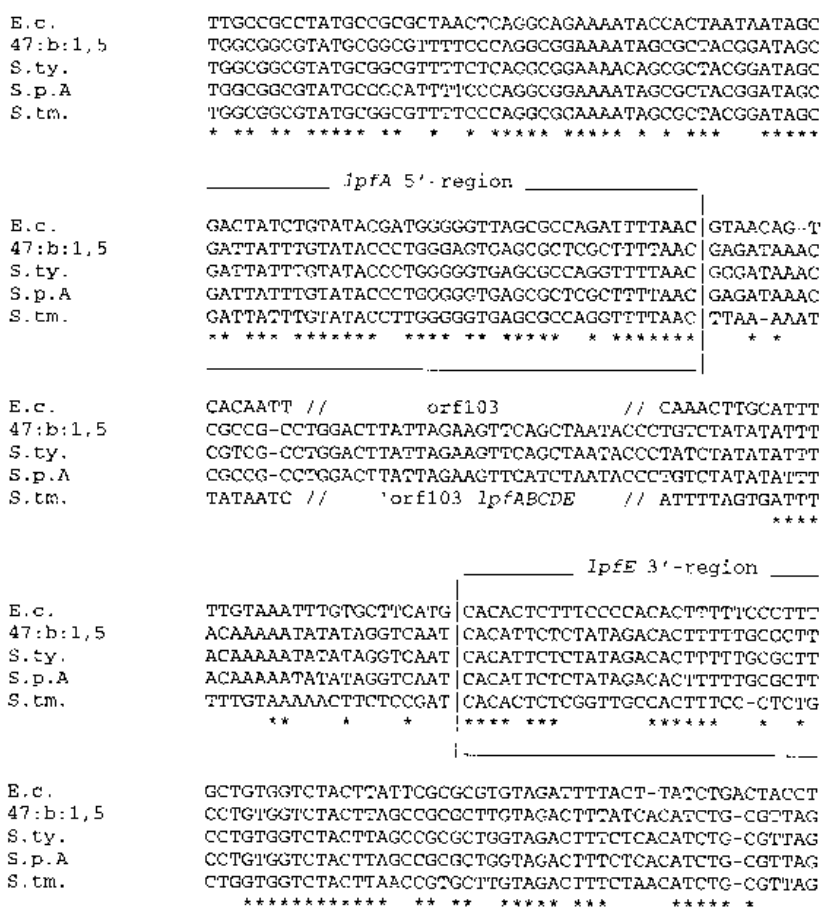

FIG. 5. Comparison of nucleotide sequences of corresponding areas from the E. coli (E.c.), the $S$. paratyphi A Pa1 (S.p.A), the $S$. typhi Tp2 (S.ty.), the Salmonella II 47:b:1,5 (47:b:1,5), and the S. typhimurium (S.tm.) chromosomes by using the program CLUSTAL. Nucleotides conserved in all five sequences are indicated by stars. Areas in the $E$. coli and the $S$. typhimurium sequences where additional genes are inserted are indicated by orf103 and 'orf103 lpfABCDE, respectively.

vided the framework necessary for investigating how the acquisition of nonhomologous genetic material has contributed to the evolution of the genus Salmonella. In this study, we determined the phylogenetic distribution of fimbrial operons among Salmonella serotypes (Fig. 2, 3) in order to resolve the genealogy of the recombinational events which led to their acquisition. The sef fimbrial operon has a limited phylogenetic distribution, indicative of recent acquisition by two distantly related evolutionary lineages within $S$. enterica subspecies I. Similarly, the phylogenetic distribution of the pef operon provides evidence that it too was introduced recently into two lineages of $S$. enterica subspecies I. The lpf operon entered the genome earlier during evolution, in a lineage ancestral to the genus Salmonella. Like the lpf operon, the fim operon is not present in E. coli or related species, and its phylogenetic distribution indicates acquisition by an ancestor of the genus Salmonella. Finally, the agf operon is the most ancient of the Salmonella fimbrial operons and was most likely already present in a common ancestor of E. coli and the genus Salmonella. This sequence of recombinational events created new evolutionary lineages of Salmonella possessing different adhesive properties. Another factor contributing to the variety of fimbrial repertoires observed among Salmonella serotypes is the loss of adhesive properties. In the case of lpf, the entire operon has been deleted in several lineages of the genus Salmonella, leading to a scattered phylogenetic distribution (Fig. $2,3)$. The comparative analysis presented here shows that horizontal gene transfer and deletion events have created unique combinations of fimbrial operons among Salmonella serotypes. Besides these mechanisms, diversity in adhesive properties is further increased by genetic changes that cannot 
be detected by hybridization analysis. For example, in the case of the fim operon encoding type 1 fimbriae, point mutations can abolish mannose-sensitive adhesion and result in expression of fimbriae with unspecified binding properties (so-called type 2 fimbriae) $(19,23,24,33,41)$.

It is not clear which selective forces led to the development of the scattered phylogenetic distribution of lpf. It has been shown that expression of the lpf fimbrial operon can be advantageous in mice (7), but it could be a disadvantage in other host species. Colonization of these other hosts could have selected for variants that lost $l p f$. A similar situation may exist for the growth of Salmonella serotypes in chickens, a habitat that appears to select against type 1 fimbriation and flagellation. For example, the avian species-adapted serotypes Salmonella gallinarum, the agent of fowl typhoid, and Salmonella pullorum, which causes pullorum disease in chicks, are nonflagellate and carry point mutations in their fim operons that abolish mannose-sensitive adhesion (19, 24, 41). Similarly, S. typhimurium strains that are nonmotile and lack type 1 fimbriation are isolated primarily from cases of avian disease (23). As is the case with $l p f$ and fim, the phylogenetic distribution of sef and pef could be seen as a result of adaptation to particular hosts. The sef operon was found to be present in two distantly related lineages within $S$. enterica subspecies I (Fig. 2). One of these phylogenetic groups included the avian species-adapted serotypes $S$. gallinarum and S. pullorum (32). The second lineage contained the common clone $S$. enteritidis En1, which is responsible for the recent worldwide increase in salmonellosis due to consumption of undercooked eggs and poultry (46). Interestingly, a sef $A$ mutant of $S$. enteritidis was impaired in its ability to colonize chicks during the first week of infection (55), suggesting that acquisition of SEF-14 fimbriae may indeed have contributed to the adaptation of Salmonella serotypes to poultry. Similarly, the pef operon is present in two distantly related evolutionary lineages (Fig. 2), one of which includes $S$. enteritidis En1 and $S$. choleraesuis Cs1, two clones with worldwide distributions that represent 93 and $88 \%$, respectively, of all isolates from these two serotypes (8). The second lineage contains strains of $S$. typhimurium. Serotypes $S$. choleraesuis and $S$. typhimurium are the most frequent isolates from pigs (48). These findings suggest that acquisition of specific fimbrial operons may have been one of the mechanisms by which Salmonella serotypes were able to expand their host range to include many domesticated animals. Because strains causing salmonellosis are usually acquired from animal sources (39), this adaptation to livestock may in turn be the reason $S$. enterica subspecies I accounts for more than $99 \%$ of human isolates.

\section{ACKNOWLEDGMENTS}

We thank S. Clegg for providing plasmids and M. W. Reeves and K. Sanderson for providing bacterial strains.

\section{REFERENCES}

1. Altschul, S. F., W. Gish, W. Miller, E. W. Myers, and D. J. Lipman. 1990. Basic local alignment search tool. J. Mol. Biol. 215:403-410.

2. Aoyama, K., A. M. Haase, and P. Reeves. 1994. Evidence for affect of random genetic drift on $\mathrm{G}+\mathrm{C}$ content after lateral transfer of fucose pathway genes to Escherichia coli. Mol. Biol. Evol. 11:829-838.

3. Ausubel, F. M., R. Brent, R. E. Kingston, D. D. Moore, J. G. Seidman, J. A. Smith, and K. Struhl (ed.). 1994. Current protocols in molecular biology. J. Wiley \& Sons, New York.

4. Bachmann, B. J. 1987. Linkage map of Escherichia coli K-12, edition 7, p. 807-876. In F. C. Neidhardt, J. L. Ingraham, K. B. Low, B. Magasanik, M. Schaechter, and H. E. Umbarger (ed.), Escherichia coli and Salmonella typhimurium: cellular and molecular biology, vol. 2. American Society for Microbiology, Washington, D.C.

5. Bäumler, A. J., and F. Heffron. 1995. Identification and sequence analysis of
lpfABCDE, a putative fimbrial operon of Salmonella typhimurium. J. Bacteriol. 177:2087-2097.

6. Bäumler, A. J., R. M. Tsolis, F. Bowe, J. G. Kusters, S. Hoffmann, and F. Heffron. 1996. The pef fimbrial operon mediates adhesion to murine small intestine and is necessary for fluid accumulation in infant mice. Infect. Immun. 64:61-68.

7. Bäumler, A. J., R. M. Tsolis, and F. Heffron. 1996. The $l p f$ fimbrial operon mediates adhesion to murine Peyer's patches. Proc. Natl. Acad. Sci. USA 93:279-283

8. Beltran, P., J. M. Musser, R. Helmuth, J. J. Farmer III, M. W. Frerichs, K. Wachsmuth, K. Ferris, A. C. McWorter, J. G. Wells, A. Cravioto, and R. K. Selander. 1988. Toward a population genetic analysis of Salmonella: genetic diversity and relationships among strains of serotypes $S$. choleraesuis, $S$. derby, S. dublin, S. enteritidis, $S$. heidelberg, $S$. infantis, $S$. newport, and $S$. typhimurium. Proc. Natl. Acad. Sci. USA 85:7753-7757.

9. Boyd, E. F., K. Nelson, F.-S. Wang, T. S. Whittam, and R. K. Selander. 1994. Molecular genetic basis of allelic polymorphism in malate dehydrogenase $(m d h)$ in natural populations of Escherichia coli and Salmonella enterica. Proc. Natl. Acad. Sci. USA 91:1280-1284.

10. Boyd, E. F., F.-S. Wang, P. Beltran, S. A. Plock, K. Nelson, and R. K Selander. 1993. Salmonella reference collection B (SARB): strains of 37 serovars of subspecies I. J. Gen. Microbiol. 139:1125-1132.

11. Buisán, M., J. M. Rodrígues-Pena, and R. Rotger. 1994. Restriction map of the Salmonella enteritidis virulence plasmid and its homology with the plasmid of Salmonella typhimurium. Microb. Pathog. 16:165-169.

12. Chiodini, R. J., and J. P. Sundberg. 1981. Salmonellosis in reptiles: a review. Am. J. Epidemiol. 113:494-499.

13. Clegg, S., S. Hull, R. Hull, and J. Pruckler. 1985. Construction and comparison of recombinant plasmids encoding type 1 fimbriae of members of the family Enterobacteriaceae. Infect. Immun. 48:275-279.

14. Clegg, S., B. K. Purcell, and J. Pruckler. 1987. Characterization of genes encoding type 1 fimbriae of Klebsiella pneumoniae, Salmonella typhimurium, and Serratia marcescens. Infect. Immun. 55:281-287.

15. Clouthier, S. C., S. K. Collinson, and W. W. Kay. 1994. Unique fimbriae-like structures encoded by sefD of the SEF14 fimbrial gene cluster of Salmonella enteritidis. Mol. Microbiol. 12:893-903.

16. Clouthier, S. C., K.-H. Müller, J. L. Doran, S. K. Collinson, and W. W. Kay. 1993. Characterization of three fimbrial genes, sefABC, of Salmonella enteritidis. J. Bacteriol. 175:2523-2533.

17. Collinson, S. K., S. C. Clouthier, J. L. Doran, P. A. Banser, and W. W. Kay. 1996. Salmonella enteritidis agf $A B C$ operon encoding thin aggregative fimbriae. J. Bacteriol. 178:662-667.

18. Collinson, S. K., S.-L. Liu, S. C. Clothier, P. A. Banser, J. L. Doran, K. E. Sanderson, and W. W. Kay. 1996. The location of four fimbrin-encoding genes, agf $A$, fim $A, \operatorname{sef} A$, and sefD, on the Salmonella enteritidis and/or $S$. typhimurium $\mathrm{Xba}-B \ln \mathrm{I}$ genomic restriction maps. Gene 169:75-80.

19. Crichton, P. B., D. E. Yakubu, D. C. Old, and S. Clegg. 1989. Immunological and genetic relatedness of type 1 and type 2 fimbriae in salmonellas of serotype Gallinarum, Pullorum and Typhimurium. J. Appl. Bacteriol. 67: 283-291.

20. Crosa, J. H., D. J. Brenner, W. H. Ewing, and S. Falkow. 1973. Molecular relationships among the salmonellae. J. Bacteriol. 115:307-315.

21. Doolittle, R. F., D. Feng, S. Tsang, G. Cho, and E. Little. 1996. Determining divergence times of the major kingdoms of living organisms with a protein clock. Science 171:470-477.

22. Doran, J. L., S. K. Collinson, J. Burian, G. Sarlos, E. C. Todd, C. K. Murno, C. M. Kay, P. A. Banser, P. I. Peterkin, and W. W. Kay. 1993. DNA-based diagnostic test for Salmonella species targeting agf $A$, the structural gene for thin, aggregative fimbriae. J. Clin. Microbiol. 31:2263-2273.

23. Duguid, J. P., E. S. Anderson, G. A. Alfredsson, R. Barker, and D. C. Old. 1975. A new biotyping scheme for Salmonella typhimurium and its phylogenetic significance. J. Med. Microbiol. 8:149-166.

24. Duguid, J. P., E. S. Anderson, and I. Campbell. 1966. Fimbriae and adhesive properties in Salmonellae. J. Pathol. Bacteriol. 92:107-137.

25. Evans, D. G., R. P. Silver, D. J. Evans, D. G. Chase, and S. L. Gorbach. 1975. Plasmid-controlled colonization factor associated with virulence in Escherichia coli enterotoxigenic for humans. Infect. Immun. 12:656-667.

26. Friedrich, M. J., N. E. Kinsey, J. Vila, and R. J. Kadner. 1993. Nucleotide sequence of a $13.9 \mathrm{~kb}$ segment of the $90 \mathrm{~kb}$ virulence plasmid of Salmonella typhimurium: the presence of fimbrial biosynthetic genes. Mol. Microbiol. 8:543-558.

27. Gulig, P. A., and R. Curtiss III. 1987. Plasmid-associated virulence of Salmonella typhimurium. Infect. Immun. 55:2891-2901.

28. Isaacson, R. E., B. Nagy, and H. W. Moon. 1977. Colonization of porcine small intestine by Escherichia coli: colonization and adhesion factors in pig enteropathogens that lack K88. J. Infect. Dis. 135:531-539.

29. Le Minor, L., and M. Y. Popoff. 1987. Designation of Salmonella enterica sp. nov., nom. rev., as the type and only species of the genus Salmonella. Int. J. Syst. Bacteriol. 37:465-468.

30. Le Minor, L., M. Y. Popoff, B. Laurent, and D. Hermant. 1986. Individualisation d'une septième sous-espèce de Salmonella: S. choleraesuis subsp. indica. subsp. nov. Ann. Microbiol. 137B:211-217. 
31. Li, J., K. Nelson, A. C. McWhorter, T. S. Whittam, and R. K. Selander. 1994. Recombinational basis of serovar diversity in Salmonella enterica. Proc. Natl. Acad. Sci. USA 91:2552-2556.

32. Li, J., H. Ochman, E. A. Groisman, E. F. Boyd, F. Solomon, K. Nelson, and R. K. Selander. 1995. Relationship between evolutionary rate and cellular location among the Inv/Spa invasion proteins of Salmonella enterica. Proc. Natl. Acad. Sci. USA 92:7252-7256.

33. Lockman, H. A., and R. Curtiss III. 1992. Isolation and characterization of conditional adherent and non-type 1 fimbriated Salmonella typhimurium mutants. Mol. Microbiol. 6:933-945.

34. Mills, D. M., V. Bajaj, and C. A. Lee. 1995. A 40kb chromosomal fragment encoding Salmonella typhimurium invasion genes is absent from the corresponding region of the Escherichia coli K-12 chromosome. Mol. Microbiol. 15:749-759.

35. Montenegro, M. A., G. Morelli, and R. Helmuth. 1991. Heteroduplex analysis of Salmonella virulence plasmids and their prevalence in isolates of defined sources. Microb. Pathog. 11:391-397.

36. Nelson, K., and R. K. Selander. 1992. Evolutionary genetics of the proline permease gene $(p u t P)$ and the control region of the proline utilization operon in populations of Salmonella and Escherichia coli. J. Bacteriol. 174: 6886-6895.

37. Nelson, K., T. S. Whittam, and R. K. Selander. 1991. Nucleotide polymorphism and evolution in the glyceraldehyde-3-phosphate dehydrogenase gene (gapA) in natural populations of Salmonella and Escherichia coli. Proc. Natl. Acad. Sci. USA 88:6667-6671.

38. Nnalue, N. A., S. Newton, and B. A. D. Stocker. 1990. Lysogenization of Salmonella choleraesuis with phage 14 increases average length of O-antigen chains, serum resistance and intraperitoneal mouse virulence. Microb. Pathog. 8:393-402.

39. O'Brien, T. F., J. D. Hopkins, J. D. Gilleece, A. A. Medeiros, R. L. Kent, B. O. Blackburn, M. B. Holmes, J. P. Reardon, J. M. Vergeront, W. L. Schell, E. Christenson, M. L. Bissett, and E. V. Morse. 1982. Molecular epidemiology of antibiotic resistance of Salmonella from animals and human beings in the United States. N. Engl. J. Med. 307:1-6.

40. Ochmann, H., and A. C. Wilson. 1987. Evolution in bacteria: evidence for a universal substitution rate in cellular genomes. J. Mol. Evol. 26:74-86.

41. Old, D. C., and S. B. Payne. 1971. Antigens of the type 2 fimbriae of Salmonellae: "cross reacting material" (CRM) of type 1 fimbriae. J. Med. Microbiol. 4:215-225.

42. Ørskov, I., F. Ørskov, H. W. Smith, and W. J. Sojka. 1975. The establishment of K99, a thermolabile, transmissible Escherichia coli $\mathrm{K}$ antigen, previously called "Kco", possessed by calf and lamb enteropathogenic strains. Acta Pathol. Microbiol. Scand. 83:31-36.
43. Popoff, M. Y., and L. Le Minor. 1992. Antigenic formulas of the Salmonella serovars, 5th ed. WHO Collaborating Center for Reference and Research on Salmonella, Institut Pasteur, Paris.

44. Reeves, M. W., G. M. Evins, A. A. Heiba, B. D. Plikaytis, and J. J. Farmer III 1989. Clonal nature of Salmonella typhi and its genetic relatedness to other salmonellae as shown by multilocus enzyme electrophoresis, and proposal of Salmonella bongori comb. nov. J. Clin. Microbiol. 27:313-320.

45. Riley, M., and A. Anilionis. 1976. Evolution of the bacterial genome. Annu. Rev. Microbiol. 32:519-560.

46. Rodrigue, D. C., R. V. Tauxe, and B. Rowe. 1990. International increase in Salmonella enteritidis: a new pandemic? Epidemiol. Infect. 105:21-27.

47. Sanger, F., S. Nicklen, and A. R. Coulson. 1977. DNA sequencing with chain-terminating inhibitors. Proc. Natl. Acad. Sci. USA 74:5463-5467.

48. Schwartz, K. J. 1991. Salmonellosis in swine. Compend. Contin. Educ. Pract. Vet. 13:139-146.

49. Selander, R. K., P. Beltran, N. H. Smith, R. M. Barker, P. B. Crichton, J. M Musser, and T. S. Whittam. 1990. Genetic population structure, clonal phylogeny, and pathogenicity of Salmonella paratyphi B. Infect. Immun. 58:1891-1901.

50. Sharp, P. M. 1991. Determinants of DNA sequence divergence between Escherichia coli and Salmonella typhimurium: codon usage, map position, and concerted evolution. J. Mol. Evol. 33:23-33.

51. Shea, J. E., M. Hensel, C. Gleeson, and D. W. Holden. 1996. Identification of a virulence locus encoding a second type III secretion system in Salmonella typhimurium. Proc. Natl. Acad. Sci. USA 93:2593-2597.

52. Stein, M. A., K. Y. Leung, M. Zwick, F. Garcia-del Portillo, and B. B. Finlay. 1996. Identification of a Salmonella virulence gene required for formation of filamentous structures containing lysosomal membrane glycoproteins within epithelial cells. Mol. Microbiol. 20:151-164.

53. Swenson, D. L., S. Clegg, and D. C. Old. 1991. The frequency of fim genes among Salmonella serovars. Microb. Pathog. 10:487-492.

54. Thorns, C. J., G. M. Sojka, I. M. Mclaren, and M. Dibb-Fuller, 1992 Characterization of monoclonal antibodies against a fimbrial structure of Salmonella enteritidis and certain other serogroup D salmonellae and their application as serotyping reagents. Res. Vet. Sci. 53:300-308.

55. Thorns, C. J., C. Turcotte, C. G. Gemmell, and M. J. Woodward. 1996 Studies into the role of the SEF14 fimbrial antigen in the pathogenesis of Salmonella enteritidis. Microb. Pathog. 20:235-246.

56. Weiss, S. H., M. J. Blaser, F. P. Paleologo, R. E. Black, A. C. McWhorter, M. A. Asbury, G. P. Carter, R. A. Feldman, and D. J. Brenner. 1986 Occurrence and distribution of serotypes of the Arizona subgroup of Salmonella strains in the United States from 1967 to 1976. J. Clin. Microbiol. 23:1056-1064. 